One-Turn Map Investigation on Space-Charge Modulation of Betatron Tune by Synchrotron Oscillation

\title{
K.Y. Ng
}

Fermi National Accelerator Laboratory, P.O. Box 500, Batavia, IL 60510

(November, 2005)

To appear in Proceedings of Workshop on High Intensity Beam Dynamics (Coulomb'05)

September 12-16, 2005, Senigallia (AN), Italy

to be published as a special issue of

Nuclear Instruments and Methods in Physics Research -Section A 


\title{
One-Turn Map Investigation on Space-Charge Modulation of Betatron Tune by Synchrotron Oscillation
}

\author{
K.Y. Ng \\ Fermi National Accelerator Laboratory, P.O. Box 500, Batavia, IL 60510
}

\begin{abstract}
The modulation of the betatron tune by the space-charge force as a result of synchrotron oscillation is studied. A one-turn-map model is devised and is applied to the various designs of the International Linear Collider (ILC) damping ring.
\end{abstract}

\section{INTRODUCTION}

The betatron space-charge tune shift experienced by a beam particle is at a maximum at the longitudinal center of the bunch. As the particle moves away from the longitudinal center under synchrotron oscillation, the spacecharge tune shift becomes smaller. Thus the betatron tune is constantly modulated by the space-charge force at at least twice the synchrotron frequency. Such modulation has been discussed by Decking and Brinkmann [1] for the TESLA damping ring. Here we wish to study the same problem using a one-turn map of the damping ring with the modulated space-charge force added at the end of each turn. This method has the merit that it is simple and fast in determining whether the synchro-betatron coupling is large or not.

\section{THEORY}

We give in this section the theory underlying the modulation and determine the various possible resonances to be encountered. Let us start from the Hamiltonian including the contribution of the normal sextupoles:

$$
H=\sum_{u=x, y}\left[\frac{p_{u}^{2}}{2}+\left(K_{u}-F_{u}^{\prime}\right) \frac{u^{2}}{2}\right]+\frac{B_{y}^{\prime \prime}}{6 B \rho}\left(x^{3}-3 x y^{2}\right) .
$$

Here, $u$ represents the horizontal/vertical displacement of the particle and $p_{u}$ the canonical momentum, $K_{u}$ is the quadrupole focusing strength, $v_{u}$ is the base tune of the lattice, $F_{u}^{\prime} u$ is the linear space-charge force to be defined later in Eq. (7), $B \rho$ is the magnetic rigidity, and $B_{y}^{\prime \prime}$ is the sextupole field. The independent variable is $s$, the distance along the ideal orbit. Because the betatron functions are defined in a linear lattice independent of beam intensity, the space-charge and the sextupole terms are treated as perturbation. Perform a canonical perturbation from $\left(u, p_{u}\right)$ to $\left(U, P_{u}\right)$ using the generating function

$$
G_{1}\left(U, p_{u} ; s\right)=\sum_{u}\left[\frac{\beta_{u}^{\prime}}{4 \beta_{0}} U^{2}-\left(\frac{\beta_{u}}{\beta_{0}}\right)^{1 / 2} U p_{u}\right]
$$

where we have deliberately introduced some reference $\beta_{0}$ so that $U=u \sqrt{\beta_{0} / \beta_{u}}$ retains the dimension of a displacement. The result of the transformation is

$$
\begin{aligned}
H= & \sum_{u} \frac{R}{2 \beta_{u}}\left(\beta_{0} P_{u}^{2}+\frac{U^{2}}{\beta_{0}}\right)-\sum_{u} \frac{R F_{u}^{\prime} \beta_{u} U^{2}}{2 \beta_{0}} \\
& +\frac{R B_{y}^{\prime \prime}}{6 B \rho}\left[\left(\frac{\beta_{x}}{\beta_{0}}\right)^{3 / 2} X^{3}-3\left(\frac{\beta_{x} \beta_{y}^{2}}{\beta_{0}^{3}}\right)^{1 / 2} X Y^{2}\right],
\end{aligned}
$$

where the independent variable has been changed to $\theta=s / R, R$ being the ring's radius, and the definition of betatron functions, $\frac{1}{2} \beta_{u} \beta_{u}^{\prime \prime}-\frac{1}{4} \beta_{u}^{\prime 2}+K_{u} \beta_{u}^{2}=1$, has been used. Next the unperturbed Hamiltonian is solved exactly in the angle-action variables $\left(\varphi_{u}, I_{u}\right)$ using the generating function

$$
G_{2}\left(a_{u}, P_{u} ; \theta\right)=\sum_{u} \frac{\beta_{0} P_{u}^{2}}{2} \cot \left(Q_{u}+\varphi_{u}\right),
$$

where $Q_{u}(\theta)=\int^{\theta} R d \theta / \beta_{u}-v_{u} \theta$ is periodic in $\theta$. We get $U=\sqrt{2 \beta_{0} I_{u}} \cos \left(Q_{u}+\varphi_{u}\right), \quad P_{u}=-\sqrt{\frac{2 I_{u}}{\beta_{0}}} \sin \left(Q_{u}+\varphi_{u}\right)$, and the Hamiltonian becomes

$$
\begin{aligned}
& H=\sum_{u} I_{u}\left\{v_{u}-\frac{1}{2} R F_{u}^{\prime} \beta_{u}\left[1+\cos 2\left(Q_{u}+\varphi_{u}\right)\right]\right\} \\
& +\frac{R B_{y}^{\prime \prime}}{6 B \rho}\left[\left(2 \beta_{x} I_{x}\right)^{3 / 2} \cos ^{3}\left(Q_{x}+\varphi_{x}\right)\right. \\
& \left.-3\left(2 \beta_{x} I_{x}\right)^{1 / 2}\left(2 \beta_{y} I_{y}\right) \cos \left(Q_{x}+\varphi_{x}\right) \cos ^{2}\left(Q_{y}+\varphi_{y}\right)\right] .
\end{aligned}
$$

Thus the betatron space-charge tune shift is $\Delta v_{u}=$ $\left\langle\frac{1}{2} R F_{u}^{\prime} \beta_{u}\right\rangle$, where $\langle\cdots\rangle$ denotes averaging over a turn.

\section{Space-Charge Tune Shift}

For convenience, we concentrate on the vertical spacecharge tune shift only and let $\Delta v_{x}=0$. This is welljustified because the the ratio of horizontal to vertical normalized rms emittances is typically $\varepsilon_{x} / \varepsilon_{y} \sim 400$ in a 
damping ring. Omitting first the modulation coming from synchrotron oscillation, the vertical linear space-charge force is given by

$$
F_{y}^{\prime} y=\frac{N_{b} r_{e}}{2 \pi \gamma^{3} \beta^{2} B} \frac{y}{\sigma_{y}\left(\sigma_{x}+\sigma_{y}\right)},
$$

where $r_{e}$ is the classical electron radius, $B=$ $\sqrt{2 \pi} \sigma_{\ell} /(2 \pi R)$ is the bunching factor, $\sigma_{\ell}$ is the rms length of the bunch containing $N_{b}$ particles, $\gamma$ and $\beta$ are the Lorentz factors, $\sigma_{x, y}$ are the rms transverse radii, and a transverse bi-Gaussian distribution has been assumed. Thus the vertical space-charge tune shift is given by

$$
\Delta v_{s c}=\left\langle\frac{R F_{y}^{\prime} \beta_{y}}{2}\right\rangle=\frac{N_{b} r_{e}}{2 \pi \gamma^{3} \beta^{2} B}\left\langle\frac{\beta_{y}}{\sigma_{y}\left(\sigma_{y}+\sigma_{x}\right)}\right\rangle .
$$

While $\sigma_{y}=\sqrt{\beta_{y} \varepsilon_{y} / \gamma \beta}$ receives contribution from the betatron oscillation amplitude, $\sigma_{x}$ receives contribution from the dispersion $D$ as well:

$$
\sigma_{x}=\sqrt{\frac{\beta_{x} \varepsilon_{x}}{\gamma \beta}+\left(\sigma_{\delta} D\right)^{2}},
$$

$\sigma_{\delta}$ being the rms momentum spread of the bunch. In many accelerators, especially hadron rings, the spacecharge tune shifts have been computed using simply

$$
\Delta v_{s c}=\frac{N_{b} r_{e} \bar{\beta}_{y}}{2 \pi \gamma^{3} \beta^{2} B \sigma_{y}\left(\sigma_{y}+\sigma_{x}\right)},
$$

where the beam radii are derived from the corresponding emittances using the average betatron functions $\bar{\beta}_{x, y}$ :

$$
\sigma_{x}=\sqrt{\frac{\bar{\beta}_{x} \varepsilon_{x}}{\gamma \beta}+\left(\sigma_{\delta} \bar{D}\right)^{2}} \text { and } \sigma_{y}=\sqrt{\frac{\bar{\beta}_{y} \varepsilon_{y}}{\gamma \beta}}
$$

with $\bar{D}=\langle D\rangle$ and $\bar{\beta}_{x, y}^{-1}=\left\langle\beta_{x, y}^{-1}\right\rangle=v_{x, y} / R$. Some properties of many designs of the ILC damping ring are listed in Table 1 . We see that the vertical space-charge tune shifts computed using the simple formula of Eq. (10) do differ significantly in many instances from the results of integrating over the lattices of the rings, Eq. (8). This is especially true in designs PPA and OCS. We find that the difference is big and the simple formula of Eq. (10) fails whenever the contribution of the dispersion to the horizontal beam size is much larger than the contribution from the horizontal betatron oscillations.

\section{Space-Charge Modulation}

The effect of synchrotron oscillation is now introduced. For a particle with maximum energy-offset excursion $\hat{E}$, the vertical space-charge tune shift is modulated by synchrotron oscillations according to

$$
\Delta v_{y}=\Delta v_{s c} e^{-\left(\hat{E} \cos v_{s} \theta\right)^{2} /\left(2 \sigma_{E}^{2}\right)},
$$

where $\sigma_{E}$ is the rms energy spread of the bunch and $v_{s}$ is the synchrotron tune. Here, $\Delta v_{s c}$ represents the maximum vertical space-charge tune shift experienced by the synchronous particle in Eq. (8). Let $t=\frac{1}{4}\left(\hat{E} / \sigma_{E}\right)^{2}$. Multipole expansion is then performed to arrive at

$$
\Delta v_{y}=\Delta v_{s c} e^{-t}\left[I_{0}(t)+\sum_{k=1}^{\infty}(-1)^{k} 2 I_{k}(t) \cos 2 k v_{s} \theta\right] \text {, }
$$

where $I_{k}(t)$ represents the modified Bessel function of order $k$. Thus the space-charge term in Eq. (6) becomes

$$
\begin{aligned}
& -\Delta v_{y} I_{y}\left[1+\cos 2\left(Q_{y}+\varphi_{y}\right)\right] \\
& =-\Delta v_{s c} I_{y} e^{-t} I_{0}(t)\left\{1+\sum_{k} 2(-1)^{k} \frac{I_{k}(t)}{I_{0}(t)} \cos 2 k v_{s} \theta\right. \\
& \left.-\cos 2\left(Q_{y}+\varphi_{y}\right)\left[1+\sum_{k} 2(-1)^{k} \frac{I_{k}(t)}{I_{0}(t)} \cos 2 k v_{s} \theta\right]\right\} .
\end{aligned}
$$

The first term in the curly brackets on the right side is a shift in the vertical betatron tune and may be included in the unperturbed Hamiltonian. The second term is the space-charge modulation of the betatron tune by the synchrotron oscillation. The third term has two parts: one is about the vertical half-integer stopband and the other is the vertical half-integer stopband under synchrotronoscillation modulation. The third term will be dropped because we are not interested in half-integer stopbands here. Thus the Hamiltonian can be simplified to

$$
H=v_{x} I_{x}+\left[v_{y}-\Delta v_{s c} e^{-t} I_{0}(t)\right] I_{y}+H_{s c}+H_{\mathrm{sex}},
$$

where

$$
\begin{aligned}
H_{s c}= & -2 \Delta v_{s c} I_{y} e^{-t} \sum_{k}(-1)^{k} I_{k}(t) \cos 2 k v_{s} \theta, \\
H_{\text {sex }}= & -\frac{R B_{y}^{\prime \prime}}{8 B \rho}\left(2 \beta_{x} I_{x}\right)^{1 / 2}\left(2 \beta_{y} I_{y}\right) \times \\
& \times\left[\cos \left(Q_{+}+\varphi_{+}\right)+\cos \left(Q_{-}+\varphi_{-}\right)\right] .
\end{aligned}
$$

In above, $Q_{ \pm}=2 Q_{y} \pm Q_{x}$ and $\varphi_{ \pm}=2 \varphi_{y} \pm \varphi_{x}$. We have also dropped the other three first-order sextupole resonant terms that do not involve the vertical betatron oscillation. Now the usual procedure is followed by an harmonic expansion in the periodic part of $H_{\text {sex }}$, leading to

$H_{\text {sex }}=-\left(2 I_{x}\right)^{1 / 2}\left(2 I_{y}\right) \times$

$\sum_{m}\left[B_{+m} \cos \left(\varphi_{+}+\beta_{+m}-m \theta\right)+B_{-m} \cos \left(\varphi_{-}+\beta_{-m}-m \theta\right)\right]$

where

$$
B_{ \pm m} e^{i \beta_{ \pm m}}=\frac{1}{8 \pi} \sum_{p} s_{p} e^{i\left(Q_{ \pm}+m \theta\right)_{p}},
$$

and the summation runs over all sextupoles at $\theta_{p}$, which are considered to be short, with strengths

$$
s_{p}=\lim _{\ell_{p} \rightarrow 0}\left(\frac{B_{y}^{\prime \prime} \ell}{2 B \rho} \beta_{x}^{1 / 2} \beta_{y}\right)_{p}
$$


TABLE 1. Some properties of the various designs of the ILC damping ring. Beam radii are computed assuming $\varepsilon_{x}=$ $8 \times 10^{-6} \pi \mathrm{m}$ and $\varepsilon_{y}=2 \times 10^{-8} \pi \mathrm{m}$.

\begin{tabular}{|c|c|c|c|c|c|c|c|}
\hline & PPA & OTW & OCS & BRU & $\mathrm{MCH}$ & DAS & TESLA \\
\hline Circumference $2 \pi R(\mathrm{~m})$ & 2824 & 3223 & 6114 & 6333 & 15935 & 17014 & 17000 \\
\hline Energy $E(\mathrm{GeV})$ & 5.00 & 5.00 & 5.066 & 3.74 & 5.00 & 5.00 & 5.00 \\
\hline Horizontal base tune $v_{x}$ & 47.810 & 45.164 & 50.84 & 65.783 & 75.783 & 83.73 & 76.31 \\
\hline Vertical base tune $v_{y}$ & 47.680 & 24.157 & 40.80 & 66.413 & 76.413 & 83.65 & 41.18 \\
\hline Momentum compaction $\left(10^{-3}\right)$ & 2.83 & 3.62 & 1.62 & 11.9 & 4.09 & 1.14 & 1.22 \\
\hline Trans./long. damping time (ms) & $20 / 10$ & $12.1 / 6.07$ & 22.2/11.1 & $25.5 / 12.8$ & $26.9 / 13.4$ & $27 / 13.5$ & $27.9 / 13.9$ \\
\hline Rf voltage (MV) & 17.76 & 21.78 & 19.27 & 23.16 & 33.7 & 48.17 & 50.00 \\
\hline $\mathrm{Rf}$ frequency $(\mathrm{MHz})$ & 500 & 714 & 650 & 650 & 650 & 500 & 497 \\
\hline Synchrotron tune $v_{S}$ & 0.0269 & 0.0418 & 0.0337 & 0.120 & 0.150 & 0.0668 & 0.071 \\
\hline Natural bunch length $\sigma_{\ell}(\mathrm{mm})$ & 6.00 & 6.00 & 6.00 & 9.00 & 9.00 & 6.00 & 6.00 \\
\hline Natural momentum spread $\sigma_{\delta}\left(10^{-3}\right)$ & 1.27 & 1.36 & 1.29 & 0.973 & 1.30 & 1.30 & 1.29 \\
\hline Particles per bunch $N_{B}\left(10^{10}\right)^{\circ}$ & 2.0 & 2.0 & 2.0 & 2.0 & 2.0 & 2.0 & 2.0 \\
\hline Average dispersion $\langle D\rangle(\mathrm{m})$ & 0.2453 & 0.0777 & 0.3227 & 0.1480 & 0.0588 & 0.0404 & 0.0153 \\
\hline \multicolumn{8}{|c|}{ Space-charge tune shift, vertical, $v_{s c}$} \\
\hline by integration & 0.0208 & 0.0447 & 0.0534 & 0.0775 & 0.139 & 0.243 & 0.273 \\
\hline by approx. formula & 0.0118 & 0.0442 & 0.0292 & 0.0757 & 0.143 & 0.241 & 0.337 \\
\hline \multicolumn{8}{|l|}{ Beam size from Eq. (11) $\sigma_{x, y}(\mu \mathrm{m})$} \\
\hline horizontal, from betatron & 87.7 & 96.4 & 124.3 & 129.4 & 165.4 & 162.6 & 170.3 \\
\hline horizontal, from dispersion & 311.5 & 105.7 & 416.3 & 144.0 & 76.5 & 52.5 & 19.7 \\
\hline total & 323.7 & 143.1 & 434.4 & 193.6 & 182.2 & 170.9 & 171.4 \\
\hline vertical, from betatron & 4.39 & 6.59 & 124.3 & 6.44 & 8.24 & 8.13 & 11.6 \\
\hline
\end{tabular}

and lengths $\ell_{p}$. In order for the sextupoles to couple with the space-charge modulation, a second-order perturbation is required. We therefore solve the Hamiltonian exactly to the first order via the canonical transformation from $\left(\varphi_{u}, I_{u}\right)$ to $\left(\phi_{u}, J_{u}\right)$. The generating function is

$$
\begin{aligned}
& G_{3}\left(\varphi_{u}, J_{u} ; \theta\right)=\varphi_{x} J_{x}+\varphi_{y} J_{y} \\
& -\left(2 J_{x}\right)^{1 / 2}\left(2 J_{y}\right) \sum_{m} \sum_{i= \pm} \frac{B_{i m}}{m-v_{i}} \sin \left(\varphi_{i}+\beta_{i m}-m \theta\right),
\end{aligned}
$$

where $v_{ \pm}=2 v_{y} \pm v_{x}$. The vertical action is transformed according to

$$
I_{y}=J_{y}-\left(2 J_{x}\right)^{1 / 2}\left(2 J_{y}\right) \sum_{i= \pm} \frac{B_{i m}}{m-v_{i}} \cos \left(\varphi_{i}+\beta_{i m}-m \theta\right) .
$$

When this is substituted into $H_{s c}$ in Eq. (16), we obtain

$$
\begin{aligned}
& H_{s c}=-2 \Delta v_{s c} J_{y} e^{-t} \sum_{k}(-1)^{k} I_{k}(t) \cos 2 k v_{s} \theta+ \\
&+\left(2 J_{x}\right)^{1 / 2}\left(2 J_{y}\right) \Delta v_{s c} e^{-t} \sum_{k, m}(-1)^{k} I_{k}(t) \sum_{i= \pm} \frac{B_{i m}}{m-v_{i}} \times \\
& \times\left[\cos \left(\phi_{i}+2 k v_{s} \theta-m \theta+\beta_{+m}\right)+\right. \\
&\left.\quad+\cos \left(\phi_{i}-2 k v_{s} \theta-m \theta+\beta_{+m}\right)\right],
\end{aligned}
$$

where $\phi_{ \pm}=2 \phi_{y} \pm \phi_{x}$. Thus to first order in sextupoles and space charge, all possible resonances are evident and they happen whenever

$$
2 \bar{v}_{y} \pm v_{x} \pm 2 k v_{s}=m
$$

is satisfied, where $\bar{v}_{y}=v_{y}-\Delta v_{s c} e^{-t} I_{0}(t)$. Near a certain resonance, we pick out that particular resonant term and the rest will drop out after averaging. Let us consider

$$
\begin{aligned}
& \langle H\rangle=v_{x} J_{x}+\bar{v}_{y} J_{y}+\frac{\alpha_{x x}}{2} J_{x}^{2}+\alpha_{x y} J_{x} J_{y}+\frac{\alpha_{y y}}{2} J_{y}^{2}+ \\
& +A_{ \pm k m} J_{x}^{1 / 2} J_{y} \cos \left(2 \phi_{y} \pm \phi_{x}+2 k v_{s} \theta-m \theta+\beta_{ \pm m}\right),
\end{aligned}
$$

where $A_{ \pm k m}=2^{3 / 2} \Delta v_{s c} e^{-t}(-1)^{k} I_{k}(t) B_{ \pm m} /\left(m-v_{ \pm}\right)$. We have included in above also the second-order amplitudedependent sextupole detunings. The resonant condition, Eq. (23), is now modified by the replacements:

$$
v_{x} \rightarrow v_{x}+\alpha_{x x} J_{x}+\alpha_{x y} J_{y}, \quad \bar{v}_{y} \rightarrow \bar{v}_{y}+\alpha_{x y} J_{x}+\alpha_{y y} J_{y} .
$$

We now transform to the frame rotating with the modulation using the generating function

$G_{4}\left(\phi_{u}, J_{1}, J_{2} ; \theta\right)=J_{1}\left[\phi_{x} \mp 2 \phi_{y} \pm\left(2 k v_{s}+m\right) \theta \mp \beta_{ \pm m}\right]+J_{2} \phi_{y}$,

where the signs are so chosen to ensure

$$
J_{1}=J_{x} \text { and } \phi_{2}=\phi_{y} .
$$

The other coordinates are

$$
\phi_{1}=2 \phi_{y} \pm \phi_{x}-\left(2 k v_{s}+m\right) \theta+\beta_{ \pm m}, \quad J_{2}=J_{y} \mp 2 J_{x} .
$$

The new Hamiltonian is

$$
\begin{aligned}
\tilde{H}= & \delta_{1} J_{1}+\frac{\alpha_{11}}{2} J_{1}^{2}+\bar{v}_{y} J_{2}+\frac{\alpha_{y y}}{2} J_{2}^{2} \\
& +A_{ \pm k m} J_{1}^{1 / 2}\left(J_{2} \pm 2 J_{1}\right) \cos \phi_{1},
\end{aligned}
$$

where the proximity of resonance is $\delta_{1}=v_{x} \pm 2 \bar{v}_{y} \pm$ $\left(2 k v_{s}-m\right)+\left(\alpha_{x y} \pm 2 \alpha_{y y}\right) J_{2}$ and $\alpha_{11}=\alpha_{x x} \pm 4 \alpha_{x y}+$ $4 \alpha_{y y}$. The Hamiltonian is now independent of $\theta$ and $\phi_{2}$; $\tilde{H}$ and $J_{2}$ are thus constants of motion which determine the particle motion completely. The difference and sum resonances are signified by $\Delta J_{y} \mp 2 \Delta J_{x}=0$. For the sum resonance, $\Delta J_{y}=2 \Delta J_{x}$ and the vertical and horizontal emittances increase without limit. For the difference resonance, the emittances are bounded with $\Delta J_{y}=-2 \Delta J_{x}$. 


\section{SINGLE-PARTICLE SIMULATION}

To see the effects of synchro-betatron resonances, we perform single-particle trackings for the various designs of the ILC damping ring. In order to incorporate sextupole contributions, a one-turn map is derived from the lattice of each ring up to the third order, [2] so that the position of the particle in the $(n+1)$ th turn can be obtained from the position in the $n$th turn via

$$
x_{i}^{(n+1)}=\sum_{j} R_{i j} x_{j}^{(n)}+\sum_{j p} T_{i j p} x_{j}^{(n)} x_{p}^{(n)}+\sum_{j p \ell} Q_{i j p \ell} x_{j}^{(n)} x_{p}^{(n)} x_{\ell}^{(n)},
$$

where the indices $i, j$ run from 1 to 6 representing the 6-dimensional position: horizontal displacement $x$, horizontal divergence $x^{\prime}$, vertical displacement $y$, vertical divergence $y^{\prime}$, longitudinal displacement $\Delta z$, and fractional momentum offset $\delta$ from the synchronous particle. The index $p$ runs from 1 to $j$ and the index $\ell$ runs from 1 to $p$.

Vertical space-charge effect is included up to the linear term by the addition of a quasi-quadrupole kick once every turn, affecting the vertical but not the horizontal. Since space-charge contributes continuously at every moment, the representation by merely a kick every turn may not be a good approximation. However, this is what we can do with the one-turn map. In any case, this can be viewed to be just a model of quadrupole modulation. Because of the rather large space-charge tune shift, we do not compute the required quadrupole strength $K_{s c}$ from the tune shift using the formula $K_{s c}=4 \pi \Delta v_{y} / \beta_{y}$, where $\beta_{y}$ is the vertical betatron function at the entry of the lattice at which the kick is to be applied. Instead, we derive the quadrupole strength from the 2-by-2 transfer matrix, which, after the kick, undergoes the change

$$
\begin{aligned}
& \left(\begin{array}{cc}
\cos \mu_{0} & \beta_{y} \sin \mu_{0} \\
-\gamma_{y} \sin \mu_{0} & \cos \mu_{0}
\end{array}\right) \\
& \longrightarrow\left(\begin{array}{cc}
\cos \mu_{0} & \beta_{y} \sin \mu_{0} \\
-\gamma_{y} \sin \mu_{0}+K_{s c} \cos \mu_{0} & \cos \mu_{0}+K_{s c} \beta_{y} \cos \mu_{0}
\end{array}\right),
\end{aligned}
$$

where $\mu_{0}$ is the vertical phase advance in a turn in the absence of space charge, and we have chosen a point of entry with $\alpha_{x}=\alpha_{y}=0$, zero dispersion, and $\beta_{y}$ at a maximum. Knowing that the phase advance is changed to $\mu=\mu_{0}-2 \pi \Delta v_{y}$, it is easy to obtain

$$
K_{s c}=\frac{2\left(\cos \mu-\cos \mu_{0}\right)}{\beta_{y} \sin \mu_{0}} .
$$

Radiation damping has not been included in the oneturn map here. However, we need to track for only one transverse damping time, because effects that show up on a time scale longer than that are irrelevant. The particle is initially at horizontal and vertical displacements of one $\sigma_{x}$ and $\sigma_{y}$ corresponding to the designed normalized rms emittances $\varepsilon_{x}=8 \times 10^{-6} \pi \mathrm{m}$ and $\varepsilon_{y}=2 \times 10^{-8} \pi \mathrm{m}$ with zero longitudinal offset and energy offset from zero to $5 \sigma_{E}$. The chromaticities of the lattices have been corrected to zero. In the numerical tracking, Eq, (12) has been used to represent the modulated tune shift $\Delta v_{y}$. Using the computed maximum vertical space-charge tune shifts listed in Table 1, we find the exhibition of resonances in all the designs except for PPA, probably because of its smallest space-charge tune shift among all the designs. Obviously, its smallest size plays an important role. Figure 1 shows the trackings for the three designs OTW, OCS, and BRU. The resonant effects for the other three designs, MCH, DAS, and TESLA are very much larger (overflows occur in computation) and are therefore not shown. Obviously, this is due to their large vertical space-charge tune shifts. The design with the next least resonant effect is the Fermilab designed OCS. We see in Fig. 1 that there is only one resonance peak with the vertical action $J_{y}$ increasing only twofold, and it occurs only at the rather large energy offset of $\sim 4.39 \sigma_{E}$.
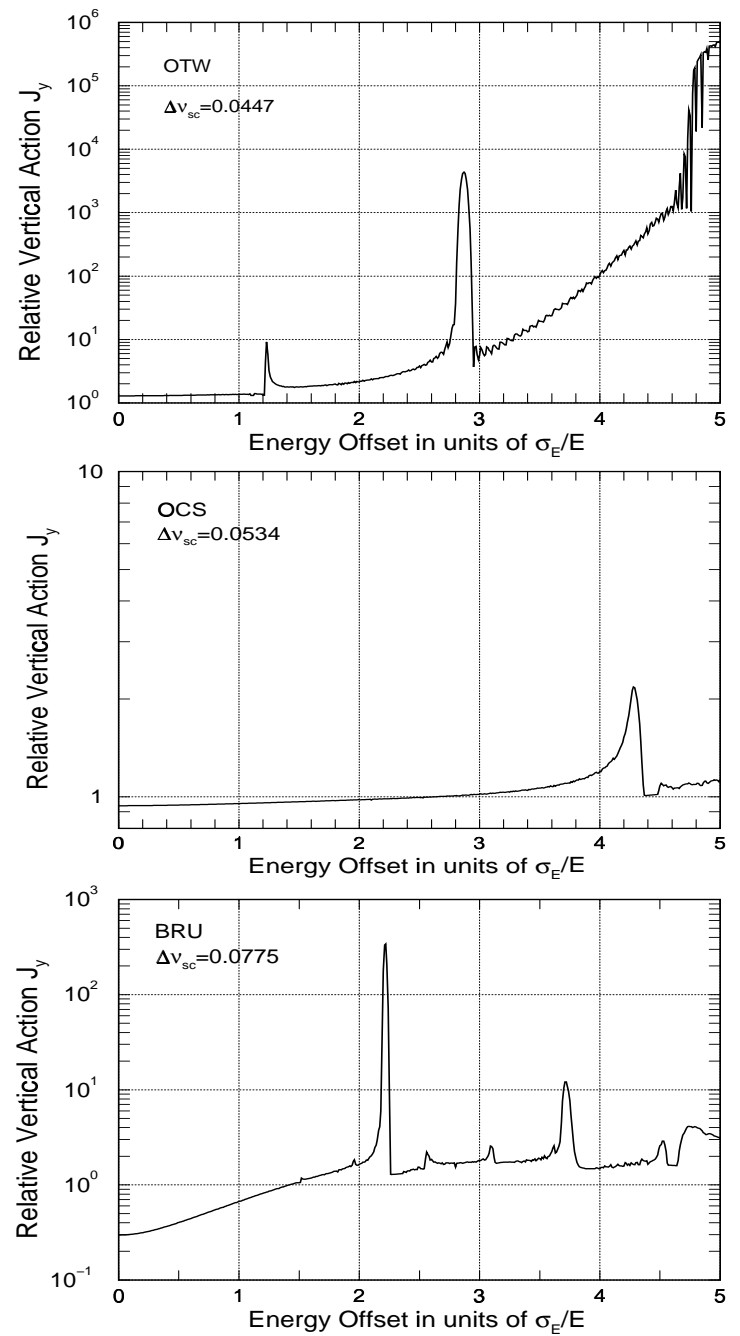

FIGURE 1. Single-particle tracking of designs OTW, OCS, and BRU of the ILC damping ring using their computed vertical space-charge tune shifts. The abscissa, maximum energy offset, is essentially the amplitude of synchrotron oscillation. 
Designs PPA and OTW are roughly of the same size, but their space-charge tune shifts have been very different, the latter is more than twice the former. This may explain why we see resonances in design OTW, but not in design PPA. Although design BRU is roughly of the same size of design OCS, the resonant effects have been very much larger. However, we have some reservation in the one-turn map of design BRU because the linear transport matrix produces horizontal and vertical betatron tune differing significantly from those obtained via MAD even in the second decimal digits. Because of this, we do not discuss design BRU any more.

Some comments of the tracking results are in order.

1. Because of the horizontal-vertical coupling driven by the sextupole fields, the horizontal and vertical actions $J_{x}$ and $J_{y}$ (or emittances) are not constants of motion. As a result, $J_{y}$ varies from turn to turn during the tracking. What is shown in Fig. 1 is the ratio of the maximum vertical action to the initial vertical action during one transverse damping time. For this reason, this ratio deviates from unity even far away from resonances. We also see that this ratio tends to increase with the energy offset of the particle. The increase remains even when zero spacecharge tune shift is substituted. This is probably due to the dependency of the Twiss parameters on energy offset, because the actions or emittances here are always computed using the Twiss parameters at the nominal energy.

2. For each design, we are very sure that the resonant effects are caused by both the sextupole contribution and the space-charge modulation driven by synchrotron oscillation. When we turn off either the synchrotron oscillation or sextupoles of the ring, all resonances disappear.

3. The contribution of quadrupole modulation, using only the $k=1$ and dc terms in Eq. (13) for $\Delta v_{y}$ instead of Eq. (12), plays only a partial role in driving the resonances. The next multipole modulation, $k=2$, contributes significantly, as may also the $k=3$ modulation. We show in Fig. 2 the contribution to design OTW of only the quadrupole modulation $(k=1)$, the $k=1$ plus $k=2$ modulations, and $k=1, k=2$, plus $k=3$ modulations. This is to be compared with the first plot in Fig. 1. We have zoomed in the region between energy offset 1 to $3 \sigma_{E}$ so that a clear comparison can be made. From the plot, it is obvious that the quadrupole modulation alone contributes very little to the resonant effects. In fact, each of the $k=2$ and $k=3$ modulations also contributes not very much. It is their sum in certain phases that adds up to the large contribution. The contributions of various order of the Bessel function are shown in Fig. 3. It is unclear why the resonances of the different multipole modulations occur at the same energy offset, in contradiction to the resonance requirement obtained in Eq. (23).

4. The actual emittance growth should be much smaller than what is depicted in Fig. 1, because the turn-to-turn change in transverse beam size has not been

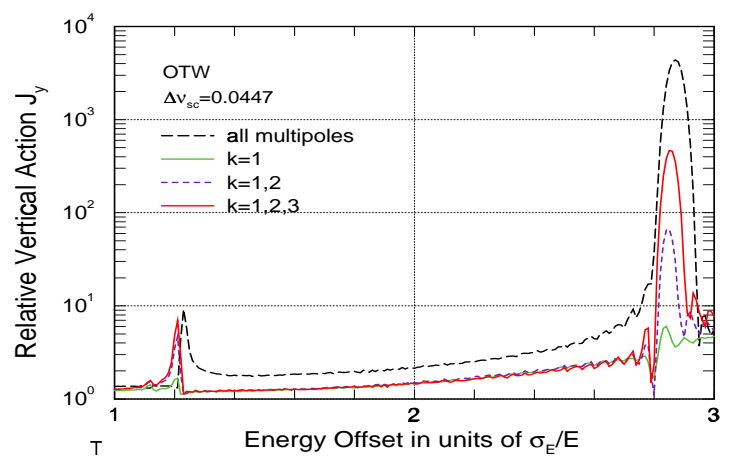

FIGURE 2. Single-particle tracking results of various multipole modulations making up the synchrotron motion are shown for design OTW in the energy offset of 1 to $3 \sigma_{E}$. It is clear that the modulations of many multipoles are required to produce the effects of the synchrotron modulation.

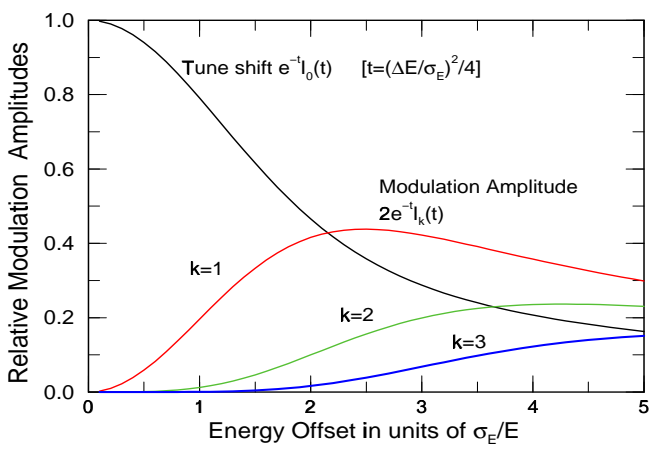

FIGURE 3. Relative strengths of multipole modulations $2 e^{-t} I_{k}(t)$ in Eq. (13) are depicted: the $k=1, k=2$, and $k=3$ modulations. The relative average amount of space-charge tune shift $e^{-t} I_{0}(t)$ is also shown.

updated during the tracking. It is interesting to point out that given sufficient time, the emittance growth reverses and the emittance goes back to zero, as if the particle has embarked on an excursion in a large island.

\section{IDENTIFICATION OF RESONANCES}

We concentrate first on the resonance of design OTW when the energy offset is $2.87 \sigma_{E}$ (see first plot of Fig. 1). The transverse emittances for one damping time ( $\sim 1100$ turns) are shown in the top two plots of Fig. 4. We see both the horizontal and vertical emittances (or actions) increase, implying that this is the sum resonance. Notice that the scales in the two plots are very different because of the much larger emittance in the horizontal than the vertical. However, we do not understand why the growth of the vertical is exponential while it is roughly linear for the horizontal. For the resonance of design OTW at $1.23 \sigma_{E}$, the emittances shown in the lower two plots of Fig. 4 also reveal a sum resonance since both emittances increase. Here, both the horizontal and vertical growths are roughly linear, with the horizontal at a 

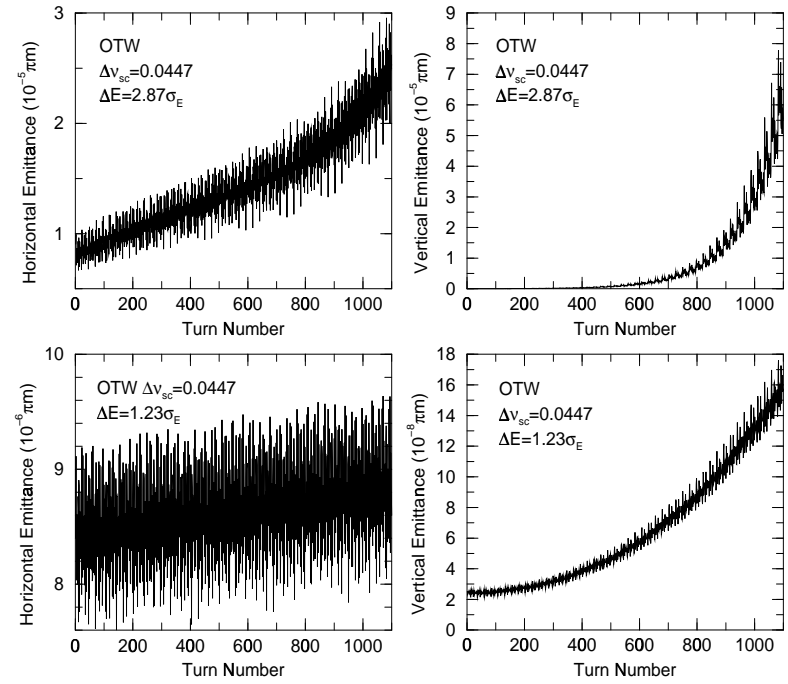

FIGURE 4. Horizontal (left) and vertical (right) emittances of a particle in the OTW bunch as functions of turn number at resonant energy-offset $2.87 \sigma_{E}$ (top) and $1.23 \sigma_{E}$ (bottom). Both are sum resonances because both horizontal and vertical emittances increase.

rate roughly ten times the rate of the vertical. The reason is unknown.

We have also analyzed the resonance of design OCS at $4.39 \sigma_{E}$ in Fig. 5. We see both horizontal and vertical emittances increase indicating the occurrence of a sum resonance. Again, we do not understand why the horizontal increases by about 10 times the vertical.
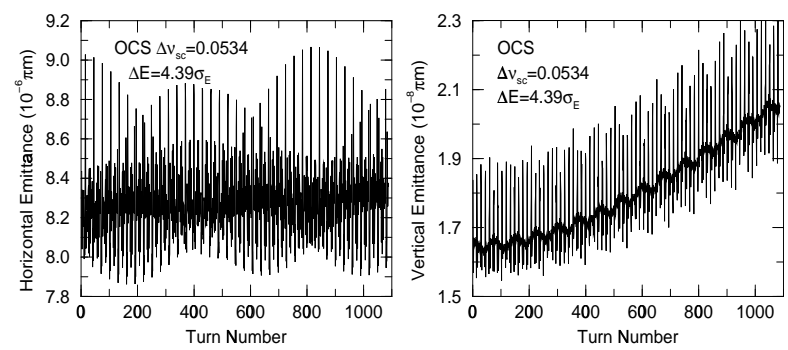

FIGURE 5. Horizontal (left) and vertical (right) emittances of a particle in the OCS bunch as functions of turn number at resonant energy-offset $4.39 \sigma_{E}$, revealing a sum resonance because both horizontal and vertical emittances increase.

Although no resonances show up with design PPA at its vertical space-charge tune shift of $\Delta v_{s c}=0.0208$, however, resonances do occur when the space-charge tune shift is increased to say 0.05 , as is illustrated in the top plot of Fig. 6, where a resonance at energy offset equal to $2.41 \sigma_{E}$ is evident. The resonance analyzed in the lower plots shows a difference resonance because the horizontal emittance decreases while the vertical increases. However, it is unclear why the horizontal emittance decreases almost linearly while the vertical emittance increases exponentially.
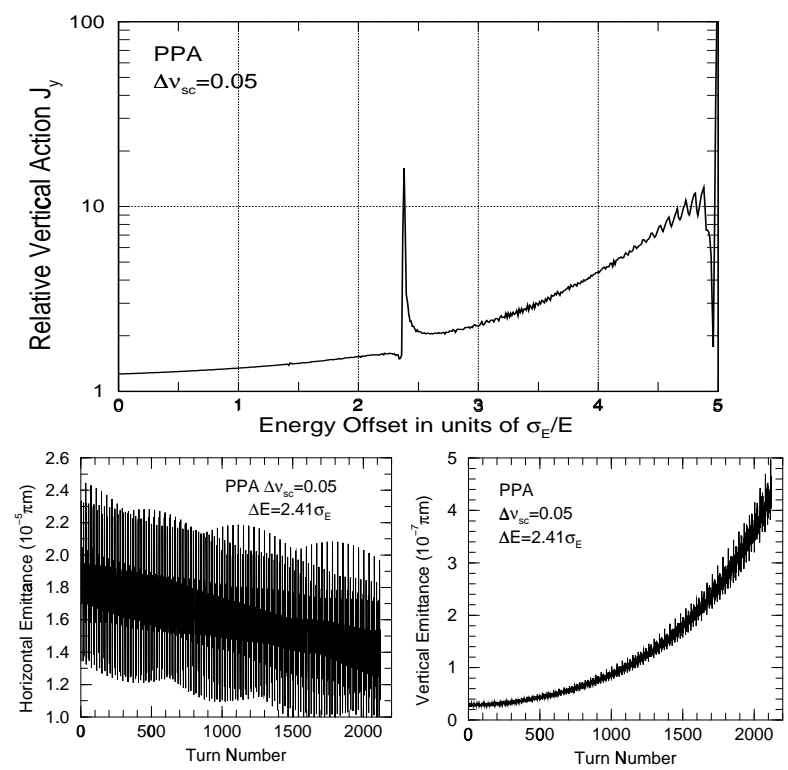

FIGURE 6. Top: Resonant effects of design PPA when the vertical space-charge tune shift is increased to $\Delta v_{s c}=0.05$. A resonance at energy offset $2.41 \sigma_{E}$ is evident. Bottom: Horizontal (left) and vertical (right) emittances of a particle in the PPA bunch as functions of turn number at the resonant energy offset $2.41 \sigma_{E}$. This is a difference resonance.

\section{CONCLUSION}

A one-turn-map model is devised to study the spacecharge modulation of the betatron tune due to synchrotron motion. Only design PPA has been found free of resonances because of its low space-charge tune shift. Resonances are contributed not only by quadrupole modulation; $k=2,3, \cdots$ modulations do play an important role. Some resonances have been identified as sum and difference resonances by monitoring the increases and decreases of the transverse emittances. However, there still remain many unanswered questions concerning the identifications. Although the representation of the spacecharge force by merely one quadrupole kick per turn is nonideal, however, the model does provide a simple way to survey resonant effects in emittance growth with negligible computer time. This model can be improved easily by including the nonlinear space-charge force and by introducing a small number of space-charge kicks per turn. Radiation damping can also be added turn by turn.

\section{REFERENCES}

1. W. Decking and R. Brinkmann, Space Charge Problems in the TESLA Damping Ring, Proc. 7th European Part. Accel. Conf. EPAC'00, ed. M. Regler (Vienna, Austria, June 26-30, 2000) p. 1024.

2. The one-turn maps were derived by L. Emery and A. Xiao using the code 'ELEGANT.' 